\title{
MONOTERPENOS DI-HIDROXILADOS E HIDRÓXI-NITROGENADOS 1,2 E 1,3 COMO LIGANTES QUIRAIS EM REAÇÃO DE REFORMATSKY ASSIMÉTRICA
}

Carlos Magno R. Ribeiro*, Cristina M. Morita e Mônica P. Maia

Departamento de Química Orgânica, Instituto de Química, Universidade Federal Fluminense, Outeiro de São João Batista, s/n, 24020-150 Niterói - RJ, Brasil

Recebido em 9/5/07; aceito em 30/8/07; publicado na web em 10/3/08

\begin{abstract}
1,2- AND 1,3- DIHYDROXYLATED AND HYDROXYNITROGENATED MONOTERPENES AS CHIRAL LIGANDS IN THE ASYMMETRIC REFORMATSKY REACTION. This study describes the use of three (-)- $\alpha$-pinene derivatives, one diol-1,2 [(-)$(1 \mathrm{R}, 2 \mathrm{R}, 3 \mathrm{~S}, 5 \mathrm{R})-2,6,6-$ trimethylbicyclo[3.1.1]heptane-2,3-diol 4] and two piridine-hydroxy derivatives [(+)-(1R,2S,3R,5S)-2,6,6trimethyl-3-(2-pyridinylmethyl)bicyclo[3.1.1] heptan-3-ol 7 and (-)-(1R,2S,3R,5S)-2,6,6-trimethyl-3-[2-(2-pyridinyl) ethyl]bicyclo[3.1.1]heptan-3-ol 8]; one diol-1,3 [(-)-(1S,2R,5S)-2-(1-hydroxy-1-methylethyl)-5-methylcyclohexanol 5] derived from (+)-isopulegol 2 and one diol-1,3 [(+)-(1R,2R,5R)-2-(1-hydroxy-1-methylethyl)-5-methylcyclohexanol 6] derived from (+)-neoisopulegol 3, as ligands in the asymmetric Reformatsky reaction. The best enantiomeric excess of $\beta$-hydroxy ester obtained in the Reformatsky asymmetric reaction was $18 \%$ using ligand $\mathbf{6}$, and the chemical yield of the reactions was $65 \%$ on average.
\end{abstract}

Keywords: Reformatsky reaction; chiral ligands; monoterpene.

\section{INTRODUÇÃO}

O controle da estereoquímica de uma molécula é um dos mais importantes temas na síntese orgânica, o que tem estimulado diversos grupos de pesquisa a preparar novos auxiliares, ligantes ou catalisadores quirais para serem usados em diversos tipos de reações visando a formação de ligações carbono-carbono. ${ }^{1}$ A reação de Reformatsky é uma reação clássica, podendo ser usada para obtenção de $\beta$-hidroxiésteres. Além disso, emprega zinco metálico, $\alpha$-haloésteres e aldeídos ou cetonas como reagentes. Os aspectos mecanísticos, condições reacionais, além de outras características desta reação têm sido amplamente discutidos em alguns artigos de revisão. ${ }^{2-4}$ Uma vez que a reação de Reformatsky pode levar à formação de pelo menos um estereocentro, tem-se utilizado substratos quirais, haloésteres quirais, auxiliares quirais ou ligantes quirais (Esquema 1) visando a obtenção de $\beta$-hidroxiésteres enantioméricamente puros. ${ }^{3,4}$

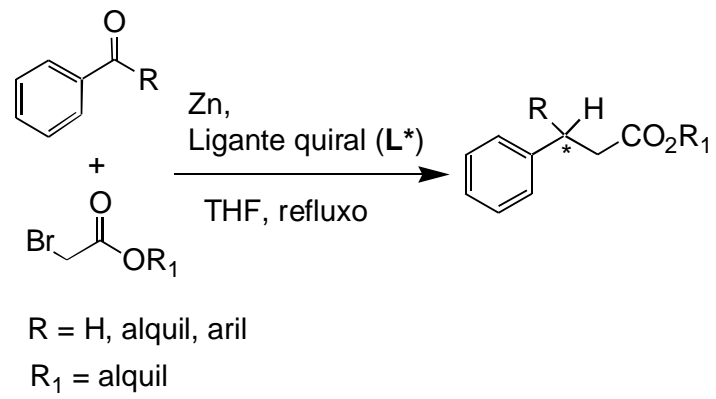

Esquema 1

Em 1972, Guetté e colaboradores empregaram pela primeira vez um ligante quiral na reação de Reformatsky, no caso, o alcalóide natural conhecido como esparteína. ${ }^{5}$ A partir de então, neste tipo de reação foram usadas como ligantes quirais algumas classes de

*e-mail: gqocmrr@vm.uff.br substâncias, tendo como foco principal derivados da efedrina. ${ }^{4,6}$ Após a publicação de um artigo de revisão sobre a aplicação de ligantes quirais em reações de Reformatsky assimétrica, ${ }^{4}$ em 2004, um trabalho discutiu o uso de derivados de carboidratos, ${ }^{7}$ um segundo apresentou os resultados obtidos com terpenos ${ }^{8}$ e outros três ainda mostraram o emprego de derivados da prolina ${ }^{9}$ e efedrina ${ }^{10}$ como ligantes quirais na reação de Reformatsky assimétrica. Lembramos que os terpenos, além de serem usados em reações de Reformatsky assimétrica, como já mencionado, também têm sido aplicados em outros tipos de reações, como, por exemplo, nas de adição de organozinco às substâncias carboniladas, ${ }^{11}$ na adição de reagentes organometálicos, $\mathrm{R}_{2} \mathrm{CuLi}$, em substâncias carboniladas $\alpha, \beta$ - insaturadas, ${ }^{12}$ nas reações aldólicas assimétricas, ${ }^{13,14}$ na hidrólise de epóxidos para a síntese de intermediários quirais ${ }^{15}$ e em reações de alquilação. ${ }^{16}$

As diaminas, os aminoálcoois e aminodióis quirais têm sido os grupos funcionais mais investigados como ligantes nas reações de Reformatsky assimétrica. Nos cerca de 30 trabalhos relatados na literatura para este tipo de reação são descritas a avaliação de aproximadamente 90 ligantes, sendo que, de forma geral, o rendimento químico tem atingido $70 \%$, e uma média de dez ligantes levou ao excesso enantiomérico na razão de 70-90\%. . $^{3-10,17,18}$

Devido ao fato de terem sido poucos os ligantes quirais que levaram à formação de $\beta$-hidroxiésteres em bons excessos enantioméricos nas reações de Reformatsky assimétrica, à baixa diversidade estrutural dos ligantes estudados para esta reação, e apenas um artigo descrever a aplicação de monoterpenos como ligantes em reações de Reformatsky assimétrica, ${ }^{8}$ acreditamos que muito se tem a averiguar sobre esse tema. Em virtude do nosso interesse na obtenção de $\beta$ hidroxiéster, ${ }^{19}$ bem como na reação de Reformatsky assimétrica, ${ }^{4,18}$ resolvemos avaliar neste tipo de reação o uso de cinco ligantes quirais derivados dos monoterpenos (-)- $\alpha$-pineno 1, (-)-isopulegol 2 e (+)neo-isopulegol 3. Decidimos usar os dióis-1,3 com substituintes cis(+)-6 e trans-(-)-5 que são produtos comerciais derivados do (-)isopulegol 3 e (+)-neo-isopulegol $\mathbf{2}$, respectivamente, enquanto que o diol-1,2 cis-(-)-4 e os dois piridinoidroxilados (+)-7 e (-)-8 são derivados do (-)- $\alpha$-pineno 1 (Figura 1). 


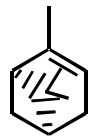

$(-)-1$

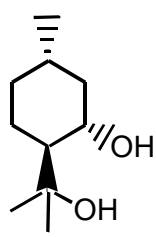

$(-)-5$

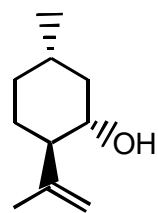

$(+)-2$

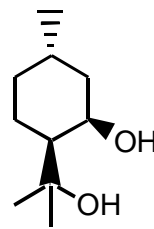

$(+)-6$

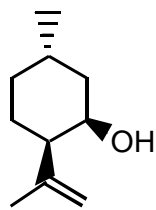

$(+)-3$

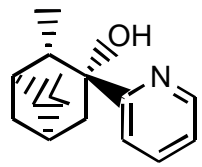

$(+)-7$

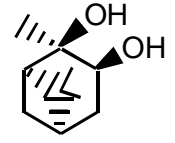

$(-)-4$
Figura 1. Ligantes derivados de monoterpenos

\section{PARTE EXPERIMETAL}

Os reagentes e solventes foram usados após purificação. ${ }^{20} \mathrm{Os}$ dióis (-)-5 e (+)-6 e os demais reagentes são comerciais (Aldrich). Os espectros de IV foram obtidos no espectrômetro FTIR SpectrumOne, Perkin-Elmer. Os espectros de RMN de ${ }^{1} \mathrm{H}$ e ${ }^{13} \mathrm{C}$ foram obtidos em um espectrômetro Varian $300 \mathrm{MHz}$, e os espectros de massas em um aparelho V. G. AutoSpec, sob impacto a $70 \mathrm{eV}$. Os fragmentos foram descritos como a relação entre as unidades de massa atômica e carga $(\mathrm{m} / \mathrm{z})$ e a abundância relativa em porcentagem. As medidas de rotação óptica foram realizadas em um polarímetro Perkin-Elmer 243 B. Os excessos enantioméricos dos $\beta$ hidroxiésteres 14-16, obtidos das reações de Reformatsky assimétrica, foram calculados a partir das áreas dos picos relativos aos hidrogênios carbinólicos dos espectros de RMN de ${ }^{1} \mathrm{H}$, usando-se reagente de deslocamento quiral $\left(\mathrm{Eu}(\mathrm{hfc})_{3}\right)$. A configuração absoluta dos $\beta$-hidroxiésteres 14-16 foi determinada por comparação dos espectros de $\mathrm{RMN}^{1} \mathrm{H}$ de padrões e daqueles adquiridos com o uso de reagente de deslocamento, conforme literatura. ${ }^{18,19}$

(-)-(1R, 2R, 3S, 5R)-2,6,6-trimetilbiciclo[3.1.1]heptano-2,3-diol $4^{21}$ [(-)-pinanodiol]: A uma solução de tetróxido de ósmio $(0,0787$ mmol) em terc-butanol (1 mL) foi adicionado (-)- $\alpha$-pineno 1 (25 $\mathrm{mmol})$, NMO (34 mmol), piridina (2 $\mathrm{mL})$, água $(15 \mathrm{~mL})$ e tercbutanol $(50 \mathrm{~mL})$. A solução púrpura foi refluxada sob atmosfera de nitrogênio durante $17 \mathrm{~h}$. A seguir, a mistura foi resfriada a $25{ }^{\circ} \mathrm{C}$, tratada com uma solução a $20 \%$ de bissulfito de sódio $(20 \mathrm{~mL})$ e concentrada a vácuo até total remoção do terc-butanol. Extração com éter etílico ( 3 x $100 \mathrm{~mL}$ ), seguida de lavagem da fase orgânica com solução saturada de $\mathrm{NaCl}$ e secagem com sulfato de sódio anidro forneceu, após evaporação, um $\mathbf{4}$ como um sólido branco $(3,95 \mathrm{~g})$ em $93 \%$ de rendimento, $[\alpha]_{\mathrm{D}}^{25}=-8,6^{\circ}$ (c. 6,5 ; tolueno). RMN de ${ }^{1} \mathrm{H}\left(300 \mathrm{MHz}, \mathrm{CDCl}_{3}\right), \delta(\mathrm{ppm}): 0,92(3 \mathrm{H}, \mathrm{s}) ; 1,25(3 \mathrm{H}, \mathrm{s})$; $1,29(3 \mathrm{H}, \mathrm{s}) ; 1,36(1 \mathrm{H}, \mathrm{d}, J=10,3) ; 1,62(1 \mathrm{H}, \mathrm{ddd}, J=14,3 ; 5,2 \mathrm{e}$ $2,4)$; 1,94-1,88 (1H, m); $1.99(1 \mathrm{H}, \mathrm{t}, J=5,9) ; 2,50-2,36(1 \mathrm{H}, \mathrm{m})$; $3,07$ (sl, OH); 3,90 (1H, dd, $J=9,4$ e $J=5,2)$. RMN de ${ }^{13} \mathrm{C}(75$ $\mathrm{MHz}, \mathrm{CDCl}_{3}$ ), $\delta$ (ppm): 23,9; 27,6; 27,8; 29,4; 37,7; 38,6; 40,2; 53,$6 ; 68,8 ; 73,5$. IV (filme), $v_{\text {máx. }} / \mathrm{cm}^{-1}: 3650-3584 ; 2990,1380,1250$, 930,890 .

(+)-(1S,2S,3S,5R)-2,6,6-trimetilbiciclo[3.1.1]heptan-3-ol 9 [(+)-isopinocanfeol]:22,23 A uma mistura de (-)- $\alpha$-pineno 1 (27,2 g; $0,02 \mathrm{~mol})$ e $\mathrm{NaBH}_{4}(3,1 \mathrm{~g} ; 0,08 \mathrm{~mol})$ em THF anidro $(120 \mathrm{~mL})$, a 0 ${ }^{\circ} \mathrm{C}$ e sobre atmosfera de nitrogênio foi adicionado gota a gota $\mathrm{BF}_{3}$. Et $\mathrm{O}_{2} \mathrm{O}(14 \mathrm{~mL})$. A mistura assim obtida foi agitada durante 45 min. $\mathrm{O}$ excesso de hidreto foi destruído por adição lenta de água gelada $(20 \mathrm{~mL})$ e $3 \mathrm{M} \mathrm{NaOH}(40 \mathrm{~mL})$. A solução foi resfriada a 0 ${ }^{\circ} \mathrm{C}$ e a ela foi adicionado lentamente $30 \% \mathrm{H}_{2} \mathrm{O}_{2}(22 \mathrm{~mL})$ de modo que a temperatura não ultrapassasse $50{ }^{\circ} \mathrm{C}$. Após a adição, a solução foi agitada vigorosamente durante $1 \mathrm{~h}$ a temperatura ambiente, extraída com éter etílico $(5 \times 100 \mathrm{~mL})$ e secada com sulfato de sódio anidro. Evaporação do solvente forneceu um resíduo, que foi destilado a pressão reduzida $\left(80-82{ }^{\circ} \mathrm{C}\right.$ a $\left.2 \mathrm{~mm} \mathrm{Hg}\right)$. O destilado, que cristaliza imediatamente, foi recristalizado em éter de petróleo levando a $9(24,3 \mathrm{~g} ; 79 \%)$ como um sólido, $[\alpha]_{\mathrm{D}}^{25}=+32,8^{\circ}$ (c. 10; tolueno). RMN de ${ }^{1} \mathrm{H}\left(300 \mathrm{MHz}, \mathrm{CDCl}_{3}\right), \delta$ (ppm): 4,05 (1H, ddd, $\left.J_{3,4 e}=4,8 ; J_{3,4 a}=9,3 ; J_{3,2}=5,2\right) ; 2,57-2,47(1 \mathrm{H}, \mathrm{m}) ; 2,41-2,33(1 \mathrm{H}$, $\mathrm{m}) ; 1,97-1,88(1 \mathrm{H}, \mathrm{m}) ; 1,79\left(1 \mathrm{H}, \mathrm{ddd}, J_{1,7 e}=6,0 ; J_{1,5}=6,0 ; J_{1,2}=\right.$ $1,8) ; 1,73\left(1 \mathrm{H}, \mathrm{ddd}, J_{4,4}=14,0 ; J_{4 a, 3}=9,2 ; J_{4 a, 5}=4,5\right) ; 1.13(3 \mathrm{H}, \mathrm{d}$, $\left.J_{10,2 a}=7,5\right) ; 1,21(3 \mathrm{H}, \mathrm{s}) ; 1,06\left(1 \mathrm{H}, \mathrm{d}, J_{7,7}=9,6\right) ; 0,92(3 \mathrm{H}, \mathrm{s}) . \mathrm{RMN}$ $\mathrm{de}^{13} \mathrm{C}\left(75 \mathrm{MHz}, \mathrm{CDCl}_{3}\right), \delta$ (ppm): 20,6;23,5; 27,5; 34,2; 38,0; 38,$9 ; 41,6 ; 47,6 ; 47,7 ; 71,5$. IV (filme), $v_{\text {máx }} / \mathrm{cm}^{-1}: 3540-3020 ; 2980$; 2895; 1450;1380; 1360; 1145; 1040; 1000; 925.

(+)-(1S,2S,5R)-2,6,6-trimetilbiciclo[3.1.1]heptan-3-ona $\mathbf{1 0}^{22,23}$ [(+)-isopinocanfona]: Em um balão bitubulado equipado com funil de adição e termômetro, foi adicionada uma solução de (+)isopinocanfeol 9 (17 g; 0,11 mol) em acetona $(23 \mathrm{~mL})$ e resfriada a $0{ }^{\circ} \mathrm{C}$. A mistura foi agitada vigorosamente durante $15 \mathrm{~min}$. A essa mistura foi adicionada, gota a gota, uma solução de $\mathrm{CrO}_{3}(7,5 \mathrm{~g})$, $\mathrm{H}_{2} \mathrm{SO}_{4}$ concentrado $(6,4 \mathrm{~mL})$ e água $(20 \mathrm{~mL})$ através do funil de adição, mantendo a temperatura entre $3-4{ }^{\circ} \mathrm{C}$. Ao término da reação, a mistura foi agitada por $2 \mathrm{~h}$ a temperatura ambiente, tratada com água $(20 \mathrm{~mL})$ e extraída com éter etílico $(3$ x $20 \mathrm{~mL})$. As fases orgânicas combinadas foram evaporadas a pressão reduzida e o resíduo obtido foi destilado a vácuo (p.e.: $69-70{ }^{\circ} \mathrm{C}$ a $5 \mathrm{~mm} \mathrm{Hg}$ ) fornecendo 10 (12,3 g; 74\%) como um óleo incolor. RMN de ${ }^{1} \mathrm{H}$ (300 MHz, $\left.\mathrm{CDCl}_{3}\right), \delta$ (ppm): 2,69-2,42 (4H,m); 2,15-2,10 (1H, m); $2,05\left(1 \mathrm{H}, \mathrm{dt}, J_{1,7 e}=6,2 ; J_{1,5}=6,3 ; J_{1,2}=1.8\right) ; 1,32(3 \mathrm{H}, \mathrm{s}) ; 1,23(3 \mathrm{H}$, $\left.\mathrm{d}, J_{10,2 a}=7,5\right) ; 1,20\left(1 \mathrm{H}, \mathrm{d}, J_{7 a, 7 e}=9,6\right) ; 0,88(3 \mathrm{H}, \mathrm{s}) . \mathrm{RMN}$ de ${ }^{13} \mathrm{C}$ (75 MHz, $\mathrm{CDCl}_{3}$ ), $\delta$ (ppm): 16,5; 21,6; 26,7; 34,1; 38,7; 38,9; 44,4; 44,$7 ; 51,0 ; 214,7$. IV (filme), $v_{\text {máx. }} / \mathrm{cm}^{-1}: 2965 ; 2920 ; 1710 ; 1465$; $1365 ; 1315 ; 1175 ; 1080 ; 1045 ; 910$.

(+)-(1R,2S,3R,5S)-2,6,6-trimetil-3-(2-piridinilmetil) biciclo[3.1.1]heptan-3-ol 7:23 A uma solução 1,6 M de $n$-BuLi em hexano $(12,5 \mathrm{~mL} ; 0,02 \mathrm{~mol})$ resfriada a $-100{ }^{\circ} \mathrm{C}$ e sob atmosfera de nitrogênio foi adicionada, gota a gota, uma solução de 2-bromo piridina ( $1,9 \mathrm{~mL} ; 0,02 \mathrm{~mol})$ em THF anidro $(125 \mathrm{~mL})$ de modo que a temperatura não ultrapassasse a faixa de $-90^{\circ}$ a $-100{ }^{\circ} \mathrm{C}$, e a mistura foi agitada a essa temperatura durante $25 \mathrm{~min}$. A essa mistura foi adicionada lentamente uma solução de (+)-isopinocanfona 10 (3,04 g; 0,02 mol) em THF anidro $(20 \mathrm{~mL})$ e agitada a temperatura ambiente durante $10 \mathrm{~min}$. Ao final da reação, foi adicionada lentamente água $(70 \mathrm{~mL})$ e a mistura foi extraída com éter etílico (3x70 mL). As fases etéreas combinadas foram secadas com sulfato de magnésio anidro, o solvente evaporado, e o resíduo assim obtido foi purificado por cromatografia "flash" em gel de sílica (5\% AcOEt em hexano como eluente) fornecendo 7 (2,31 g; 50\%) como um sólido (p.f.: $74-75{ }^{\circ} \mathrm{C}$ ) $[\alpha]^{25}{ }_{\mathrm{D}}=+9,2^{\circ}$ (c. 3,04; $\mathrm{CHCl}_{3}$ ). $\mathrm{RMN}$ de ${ }^{1} \mathrm{H}\left(300 \mathrm{MHz}, \mathrm{CDCl}_{3}\right), \delta(\mathrm{ppm}): 8,46\left(1 \mathrm{H}, \mathrm{d}, J_{15,14}=5,0\right)$; $7,73\left(1 \mathrm{H}\right.$, ddd, $\left.J_{13,14}=7,3 ; J_{13,12}=8,1 ; J_{13,15}=2,0\right) ; 7,57(1 \mathrm{H}, \mathrm{dt}$, $\left.J_{12,13}=8,1 ; J_{12,14}=0,9 ; J_{12,15}=0,9\right) ; 7,16\left(1 \mathrm{H}, \mathrm{ddd}, J_{14,15}=5,0 ; J_{15,13}\right.$ $\left.=7,3 ; J_{14,12}=0,9\right) ; 5,72(\mathrm{sl}, \mathrm{O}-\mathrm{H}) ; 2,64\left(1 \mathrm{H}, \mathrm{dd}, J_{4 e, 4 a}=15,2 ; J_{4 e, 5}=\right.$ $3,3) ; 2,51-2,42(2 \mathrm{H}, \mathrm{m}) ; 2,25\left(1 \mathrm{H}, \mathrm{dq}, J_{2,10}=7,5 ; J_{2,1}=2,1\right) ; 2,11-$ $2,05(1 \mathrm{H}, \mathrm{m}) ; 1,90\left(1 \mathrm{H}, \mathrm{dt}, J_{1,7 e}=5,8 ; J_{1,2}=2,1\right) ; 1,35\left(1 \mathrm{H}, \mathrm{d}, J_{7 a, 7 e}=\right.$ 9,9); 1,38 (3H, s); 1,27 (3H, s); 1,02 (3H, d, $\left.J_{10,2}=7,5\right)$. RMN de ${ }^{13} \mathrm{C}\left(75 \mathrm{MHz}, \mathrm{CDCl}_{3}\right), \delta$ (ppm): 15,9; 21,5; 28,4; 32,3; 38,4; 41,6; 44,$7 ; 47,9 ; 49,4 ; 71,3 ; 119,5 ; 121,2 ; 137,2 ; 146,3 ; 170,0$. IV (fil- 
me), $v_{\text {máx }} / \mathrm{cm}^{-1}: 3600-3200 ; 3040 ; 2970 ; 2890 ; 2850 ; 1560 ; 1520$; $1390 ; 1140 ; 1040 ; 1030 ; 985 ; 965 ; 760$. EIMS $\mathrm{m} / \mathrm{z}(\%):[\mathrm{M}]^{+}$ 121(100); 78(46); 79(46); 122(32); 106(31).

(-)-(1R,2S,3R,5S)-2,6,6-trimetil-3-[2-(2-piridinil)etil] biciclo[3.1.1] heptan-3-ol 8: ${ }^{23}$ A uma solução 1,6 M de n-BuLi em hexano $(31,3 \mathrm{~mL} ; 0,05 \mathrm{~mol})$ sob atmosfera de nitrogênio foi adicionada, gota a gota, $\alpha$-picolina $(4,9 \mathrm{~mL} ; 0,05 \mathrm{~mol})$ e a mistura foi agitada por $1 \mathrm{~h}$ a temperatura ambiente, apresentando uma coloração vermelho. A mistura foi resfriada a $-15{ }^{\circ} \mathrm{C}$ e uma solução de (+)-isopinocanfona 10 (7,6 g; 0,05 mol) em THF anidro (50 mL) foi adicionada lentamente durante um período de $40 \mathrm{~min}$, quando a coloração diminui. Após 30 min sob agitação, foram adicionadas lentamente água $(100 \mathrm{~mL})$ e solução saturada de $\mathrm{NaCl}(100 \mathrm{~mL})$. As fases foram separadas, e a fase aquosa foi extraída com diclorometano $(3 \times 100 \mathrm{~mL})$. Os extratos orgânicos foram combinados, a seguir secado com sulfato de magnésio anidro e evaporado a vácuo, fornecendo um resíduo, que foi purificado por cromatografia "flash" em gel de sílica (5\% AcOEt em $n$-hexano como eluente) fornecendo $8(5,9 \mathrm{~g} ; 48 \%)$ como um óleo amarelo $[\alpha]^{25}{ }_{\mathrm{D}}=-48,9^{\circ}$ (c. 3,19; $\left.\mathrm{CHCl}_{3}\right) . \mathrm{RMN}$ de ${ }^{1} \mathrm{H}\left(300 \mathrm{MHz}, \mathrm{CDCl}_{3}\right), \delta$ (ppm): 8,49 $\left(1 \mathrm{H}, \mathrm{d}, J_{16,15}=5,0\right) ; 7,63\left(1 \mathrm{H}, \mathrm{dt}, J_{14,13}=7,8 ; J_{14,15}=7,8 ; J_{14,16}=1,9\right)$; $7,20-7,13(1 \mathrm{H}, \mathrm{m}) ; 7,14\left(1 \mathrm{H}, \mathrm{d}, J_{13,14}=7,8\right) ; 3,07\left(1 \mathrm{H}, \mathrm{d}, J_{11 a, 11 b}=\right.$ $14,4) ; 2,91\left(1 \mathrm{H}, \mathrm{d}, J_{11 b, 11 a}=14,4\right) ; 2,32-2,21(2 \mathrm{H}, \mathrm{m}) ; 2,15-2,04$ $(2 \mathrm{H}, \mathrm{m}) ; 1,94-1,88(1 \mathrm{H}, \mathrm{m}) ; 1,80\left(1 \mathrm{H}, \mathrm{dt}, J_{1,7 e}=5,7 ; J_{1,2}=2,7\right) ; 1,18$ $(3 \mathrm{H}, \mathrm{s}) ; 1,17(3 \mathrm{H}, \mathrm{s}) ; 1,04\left(1 \mathrm{H}, \mathrm{d}, J_{7 a, 7 e}=9,6\right) ; 0,78\left(3 \mathrm{H}, \mathrm{d}, J_{10,2}=\right.$ 7,5). RMN de ${ }^{13} \mathrm{C}\left(75 \mathrm{MHz}, \mathrm{CDCl}_{3}\right), \delta(\mathrm{ppm}): 17,2 ; 22,1 ; 27,5$; 30,$7 ; 38,1 ; 41,4 ; 45,1 ; 46,5 ; 48,2 ; 55,8 ; 70,4 ; 121,5 ; 124,7 ; 136,5$; 147,$9 ; 159,7$. IV (filme), $v_{\text {máx.od }} / \mathrm{cm}^{-1}: 3680-3100 ; 2880 ; 1585 ; 1565$; $1465 ; 1430 ; 1380 ; 1275 ; 1210 ; 1040 ; 875 ; 830 ; 765 ; 745$. EIMS $m /$ $z(\%):[\mathrm{M}]^{+}$93(100); 135(55); 162(30).

\section{Procedimento geral para a reação de Reformatsky as- simétrica}

Uma solução dos aldeídos $\mathbf{1 1}$ - $\mathbf{1 3}$ (1 mmol), com o ligante quiral $\left(\mathbf{L}^{*} ; \mathbf{4}\right.$ - 8; $\left.1 \mathrm{mmol}\right)$ e $\alpha$-bromoacetato de etila $(3 \mathrm{mmol})$ em THF anidro ( $5 \mathrm{~mL})$, é adicionada a uma solução heterogênea de zinco em pó (3 mmol, previamente ativado - lavado com solução aquosa de $\mathrm{HCl}$ e acetona e posterior secagem a $100{ }^{\circ} \mathrm{C}$ por $30 \mathrm{~min}$ ) em $\mathrm{THF}$ anidro $(10 \mathrm{~mL})$. A solução resultante foi refluxada nos tempos indicados na Tabela 1. A reação foi acidificada com $\mathrm{HCl} 10 \%$ e a mistura foi extraída com éter etílico. O extrato orgânico foi lavado com solução aquosa de $\mathrm{NaHCO}_{3}$ água e depois secado com $\mathrm{MgSO}_{4}$ e posteriormente concentrado sob pressão reduzida. $\mathrm{O}$ extrato bruto foi purificado por cromatografia de coluna em sílica gel utilizando hexano:acetato de etila 3\% como eluente. Os rendimentos químicos das reações, bem como os excessos enantioméricos dos hidroxiésteres obtidos, estão apresentados na Tabela 1.

3-hidróxi-3-fenilpropanoato de etila 14: ${ }^{18,19} \mathrm{RMN}$ de ${ }^{1} \mathrm{H}(300$ $\left.\mathrm{MHz}, \mathrm{CDCl}_{3}\right), \delta$ (ppm): 1,27 (3H, t, $\left.J=7,2\right) ; 2.70(1 \mathrm{H}, \mathrm{dd}, J=16.4$ e 4.8); 2,77 (1H, dd, $J=16,4$ e 8,4); 3,27 (br s, $-\mathrm{OH}) ; 4,18(2 \mathrm{H}, \mathrm{q}$, $J=7,2) ; 5,13$ ( $1 \mathrm{H}$, dd, $J=8,4$ e 4,8); 7,26-7,40 (5H, m). RMN de ${ }^{13} \mathrm{C}\left(75 \mathrm{MHz}, \mathrm{CDCl}_{3}\right), \delta(\mathrm{ppm}): 14,0 ; 43,2 ; 60,8 ; 70,2 ; 125,6 ; 127,7$; 128,$4 ; 142,4 ; 172,3$. IV (filme), $v_{\text {máx }} / \mathrm{cm}^{-1}: 3450(\mathrm{OH}) ; 1720(\mathrm{CO})$; 1190(CC). EIMS m/z (\%): [M] ${ }^{+}$194(32), 107(100), 79(59).

3-hidróxi-3-fenilbutanoato de etila 15: ${ }^{18,19} \mathrm{RMN}$ de ${ }^{1} \mathrm{H}(300$ $\left.\mathrm{MHz}, \mathrm{CDCl}_{3}\right), \delta(\mathrm{ppm}): 1,13(3 \mathrm{H}, \mathrm{t}, J=7,2) ; 1,54(3 \mathrm{H}, \mathrm{s}) ; 2,79$ $(1 \mathrm{H}, \mathrm{d}, J=15.9) ; 2,98(1 \mathrm{H}, \mathrm{d}, J=15,9) ; 4,06$ (2H, q, $J=7,2) ; 4,41$ (br,s, OH); 7,20-7,47 (5H, m). RMN de ${ }^{13} \mathrm{C}\left(75 \mathrm{MHz}, \mathrm{CDCl}_{3}\right), \delta$ (ppm): 13,8; 30,05; 46,3; 60,6; 72,6; 124,3; 126,7; 128,1; 146,7; 172,6 . IV (filme), $v_{\text {máx }} / \mathrm{cm}^{-1}: 3480 ; 1720 ; 1200$. EIMS $\mathrm{m} / z$ (\%): $[\mathrm{M}]^{+}$209(7), 121(100), 105(77).

3-ciclohexil-3-hidróxi-3-fenilpropanoato de etila 16: ${ }^{18,19} \mathrm{RMN}$ de ${ }^{1} \mathrm{H}\left(300 \mathrm{MHz}, \mathrm{CDCl}_{3}\right), \delta$ (ppm): 0,97-1,77 (12H, m); 1,03 $(3 \mathrm{H}$, $\mathrm{t}, J=7,2) ; 2.85(1 \mathrm{H}, \mathrm{d}, J=15,6) ; 3,01(1 \mathrm{H}, \mathrm{d}, \mathrm{J}=15,6) ; 3,96(2 \mathrm{H}$, $\mathrm{q}, J=7.2) ; 7,18-7,49(5 \mathrm{H}, \mathrm{m})$. RMN de ${ }^{13} \mathrm{C}\left(75 \mathrm{MHz}, \mathrm{CDCl}_{3}\right), \delta$ (ppm): 13,7; 26,2; 26,9; 42,2; 48,6; 60,4; 77,1; 125,7; 126,5; 127,6; 144,9; 173,4. IV (filme), $v_{\text {máx. }} / \mathrm{cm}^{-1} 3480,1720 ; 1200$. EIMS $\mathrm{m} / z$ $(\%):[\mathrm{M}]^{+.}$276(M+), 193(95), 105(100).

\section{RESULTADOS E DISCUSSÃO}

Visando o estudo da reação de Reformatsky assimétrica, inicialmente foram preparados os ligantes (-)-4, (+)-7 e (-)-8. O (-)-pinanodiol 4 foi preparado através de uma reação de oxidação do (-)- $\alpha$-pineno $\mathbf{1}$, utilizando uma solução de tetróxido de ósmio, NMO e piridina, água e terc-butanol. ${ }^{12}$ Os derivados hidróxi-nitrogenados (+)-7 e (-)-8 foram preparados de acordo com a literatura. ${ }^{23,24} \mathrm{~A}$ reação de hidroboração diastereosseletiva do (-)- $\alpha$-pineno 1 forneceu o (+)-isopinocanfeol $\mathbf{9}$, que seguido de oxidação pelo uso de $\mathrm{CrO}_{3} / \mathrm{H}_{2} \mathrm{SO}_{4}$ a $0{ }^{\circ} \mathrm{C}$ levou à formação de (+)-isopinocanfona 10. O hidróxi-nitrogenado-1,2 (+)-7 foi preparado pela adição nucleofílica de 2-litio-piridina à cetona (+)10, em THF anidro e a temperatura de $-100{ }^{\circ} \mathrm{C}$. De maneira semelhante, ao utilizar a (+)-isopinocanfona $\mathbf{1 0}$ com o ânion da picolina em THF a temperatura ambiente levou ao hidróxi-nitrogenado-1,3 (-)-8 (Esquema 2). ${ }^{21-24}$

A seguir foram efetuadas as reações de Reformatsky assimétrica reagindo-se benzaldeído 11 ou as cetonas 12 e 13 (1 mmol) com o reagente de Reformatsky RR (3 mmol; 2-bromoetilacetato de etila e zinco em pó) na presença dos ligantes quirais 4-8 $\left(\mathbf{L}^{*}\right)$, em refluxo de THF (Esquema 3).

Os resultados obtidos nestas reações estão apresentados na Tabela 1 .

Como observado na Tabela 1, os rendimentos químicos das reações podem ser considerados de razoáveis a bons, entretanto, os

Tabela 1. Reações de Reformatsky assimétrica na presença dos ligantes 4 - 8

\begin{tabular}{|c|c|c|c|c|c|c|c|c|}
\hline Entrada & $\begin{array}{l}\text { Ligante } \\
\left(\mathbf{L}^{*}\right)\end{array}$ & $\begin{array}{l}\text { Substrato } \\
\text { (S) }\end{array}$ & $\begin{array}{c}\text { Razão molar } \\
\text { S:RR:L* }\end{array}$ & $\begin{array}{l}\text { Tempo de } \\
\text { reação (h) }\end{array}$ & $\begin{array}{l}\text { Produto } \\
\text { de reação }\end{array}$ & $\begin{array}{c}\text { Rendimento } \\
(\%)^{\mathrm{a}}\end{array}$ & $\%$ e.e. $^{\mathrm{b}}$ & Configuração \\
\hline 1 & $(-)-4$ & 11 & $1: 3: 1$ & 1,5 & 14 & 63 & 7 & $\mathrm{~S}$ \\
\hline 2 & $(-)-4$ & 12 & $1: 3: 1$ & 3,0 & 15 & 92 & 5 & $\mathrm{~S}$ \\
\hline 3 & $(-)-4$ & 13 & $1: 3: 1$ & 7,5 & 16 & 37 & 0 & - \\
\hline 4 & $(-)-5$ & 11 & $1: 3: 1$ & 2,0 & 14 & 42 & 0 & - \\
\hline 5 & $(+)-6$ & 11 & $1: 3: 1$ & 1,5 & 14 & 35 & 18 & $\mathrm{~S}$ \\
\hline 6 & $(+)-7$ & 11 & $1: 3: 1$ & 1,5 & 14 & 52 & 8 & $\mathrm{R}$ \\
\hline 7 & $(-)-8$ & 11 & $1: 3: 1$ & 1,0 & 14 & 51 & 15 & $\mathrm{R}$ \\
\hline 8 & $(-)-8$ & 12 & $1: 3: 1$ & 2,5 & 15 & 41 & 3 & $\mathrm{R}$ \\
\hline
\end{tabular}

${ }^{a}$ Rendimento químico após purificação em coluna cromatográfica de sílica gel. ${ }^{\mathrm{b}}$ Determinado por $\mathrm{RMN}{ }^{1} \mathrm{H}$ com $\mathrm{Eu}(\mathrm{hfc})_{3}$. 

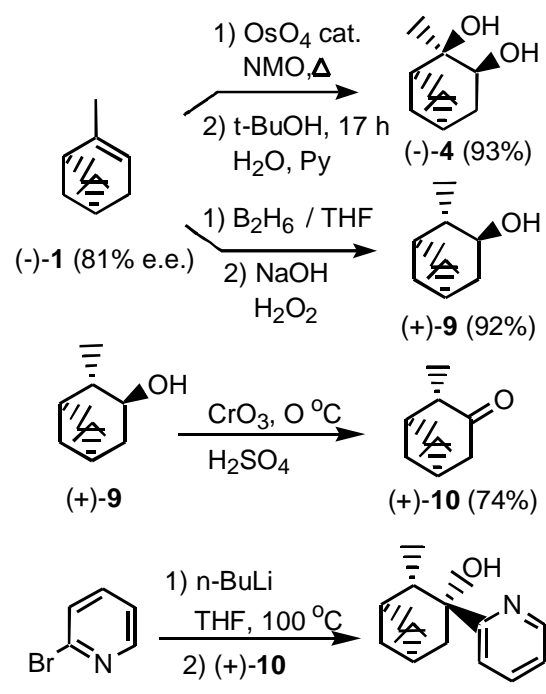

$(+)-7(50 \%)$

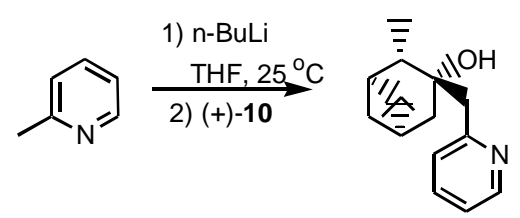

$(-)-8(48 \%)$

Esquema 2

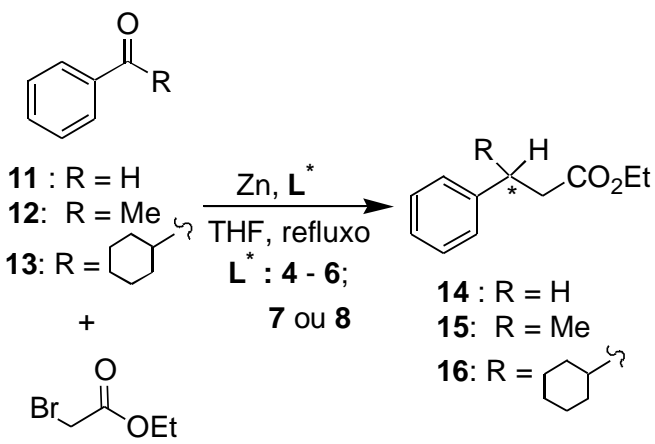

Esquema 3

excessos enantioméricos não foram os desejados. Porém, também é possível observar que o diol-1,3 (+)-6, com os grupamentos cis entre si, apresentou o melhor excesso enantiomérico que o seu isômero trans-(-)-5 (entradas 5 e 4, respectivamente), demonstrando que os derivados di-hidroxilados-1,3 com os grupamentos em cis entre si, em sistemas cíclicos, podem apresentam melhores resultados que os dióis- 1,3 com os substituintes trans. Ao se comparar os resultados de excessos enantioméricos entre o diol-1,2 (-)-4 e os dióis-1,3 (-)-5 e (+)-6, observa-se que, dependendo do arranjo estrutural, dióis-1,3 podem levar a melhores resultados que dióis1,2, no caso, o diol-1,3 cis (+)-6 e o diol-1,2 (-)-4 (entradas 5 e 1, respectivamente). Este fato, observado pela primeira vez em reações de Reformatsky assimétrica, demonstra que o espaçamento entre as hidroxilas em dióis pode contribuir na melhoria do excesso enantiomérico do produto de reação. A literatura menciona que neste tipo de reações dióis-1,2 levam a enantiosseletividades piores que os hidroxinitrogenados-1,2 com estruturas semelhantes; ${ }^{3,4}$ o mesmo foi observado ao avaliarmos o diol-1,2 (-)-4 e o hidróxinitrogenado-1,2 (+)-7 (entradas 1 e 6). Ao se comparar os hidróxinitrogenados-1,2 e 1,3, (+)-7 e (-)-8, respectivamente, conclui-se

que o espaçamento de um carbono também pode contribuir com a melhoria do excesso enantiomérico reacional (entradas 6 e 7, respectivamente). Observou-se ainda que o excesso enantiomérico do produto de reação diminui quando cetonas são utilizadas em comparação com o benzaldeído, principalmente aquelas com grupos mais volumosos (entradas 1-3 e 7 e 8 , respectivamente). Lembramos que esta é a primeira vez que se avalia a utilização de dióis e hidróxi-nitrogenados-1,3 neste tipo de reação.

\section{CONCLUSÕES}

Neste trabalho foi possível apresentar o estudo da influência de ligantes derivados dos monoterpenos (-)- $\alpha$-pineno 1, (-)isopulegol 2 e (+)-neo-isopulegol 3, em reações de Reformatsky assimétrica. Os rendimentos químicos das reações de Reformatsky assimétrica foram de razoáveis a bons, porém os excessos enantioméricos não podem ser considerados bons. Entretanto, pela primeira vez foi empregado o uso de dióis-1,3 e hidróxinitrogenados-1,3 como ligantes em reações de Reformatsky assimétrica. Foi possível avaliar que dióis-1,3, dependendo da estrutura, podem apresentar resultados melhores que dióis-1,2. O espaçamento entre as hidroxilas pode auxiliar em uma melhora da enantiosseletividade da reação. Em relação aos dióis-1,3, aqueles que apresentam grupos cis entre si em sistemas cíclicos podem levar a melhores resultados que o seu respectivo isômero trans. Ao se comparar os ligantes hidróxi-nitrogenados (+)-7 e (-)-8, observou-se também que o espaçamento entre os heteroátomos pode favorecer na enantiosseletividade da reação. Os resultados continuam a indicar que cetonas levam a piores resultados de enantiosseleção que aldeídos.

\section{AGRADECIMENTOS}

Ao Departamento de Química Orgânica da UFF pela colaboração na execução deste trabalho. Ao Prof. Dr. S. Pinheiro do Departamento de Química Orgânica da UFF por ter colaborado com a preparação do diol-1,2 (-)-4, os hidróxi-nitrogenados-1,2 e 1,3, (+)7 e (-)-8. À FAPERJ pelo financiamento.

\section{REFERÊNCIAS}

1. Nair, V.; Ros, S.; Jayan, C. N.; Pillai, B. S.; Tetrahedron 2004, 60, 1959; Orsini, F.; Lucci, E. M.; Tetrahedron Lett. 2005, 46, 1909; Buriez, O.; Durandetti, M.; Périchon, J.; J. Electroanal. Chem. 2005, 578, 63; Castro, H. F.; Anderson, W. A.; Quim. Nova 1996, 18, 544

2. Heathcok, C. H. Em Asymmetric Synthesis; Morrison, J. D., ed.; Academic Press: Orlando, 1984, vol. 3, cap. 2; Furstner, A.; Synthesis 1989, 571; Chattopadhyay, A.; Salaskar, A.; Synthesis 2000, 561.

3. Ocampo, R.; Dolbier Jr., W. R.; Tetrahedron 2004, 31, 9325.

4. Ribeiro, C. M. R.; Farias, F. M. C.; Mini-Rev. Org. Chem. 2006, 3, 1.

5. Guetté, M.; Guetté, J. P.; Capillon, J.; Tetrahedron Lett. 1971, 11, 2863; Guetté, M.; Capillon, J.; Guetté, J. P.; Tetrahedron 1973, 29, 3659.

6. Andrés, J. M.; Pedrosa, R.; Perez-Encabo, A.; Tetrahedron 2000, 56, 1217; Mi, A.; Wang, Z.; Zhang, J.; Jiang, Y.; Synth. Commun. 1997, 27, 1469; Andrés, J. M.; Martin, Y.; Pedrosa, R.; Perez-Encabo, A.; Tetrahedron 1997, 53, 3787; Pini, D.; Uccello-Barretta, G.; Mastantuono, A.; Salvadori, P.; Tetrahedron 1997, 53, 6065; Mi, A.; Wang, Z.; Chen, Z.; Jiang, Y.; Tetrahedron: Asymmetry 1995, 6, 2641; Mastantuono, A.; Pini, D.; Rolfini, C.; Salvadori, P.; Chirality 1995, 7, 499; Pini, D.; Mastantuono, A.; Salvadori, P.; Tetrahedron: Asymmetry 1994, 5, 1875; Soai, K.; Oshiro, A.; Saito, T.; J. Chem. Soc., Chem. Commun. 1993, 811; Soai, K.; Kawase, Y.; Tetrahedron: Asymmetry 1991, 2, 781.

7. Emmerson, D. P. G.; Hems, W. P.; Davis, B. G.; Tetrahedron: Asymmetry 2005, 16, 213

8. Kloetzing, R. J.; Thaler, T.; Knochel, P.; Org. Lett. 2006, 8, 1125.

9. Shin, E-K.; Kim, H. J.; Kim, Y.; Kim, Y.; Park, Y. S.; Tetrahedron Lett. 2006, 47, 1933.

10. Cozzi, P. G.; Rivalta, E.; Pure Appl. Chem. 2006, 78, 287.; Cozzi, P. G.; 
Angew.Chem., Int. Ed. 2006, 45, 2951.

11. Pu, L.; Yu, H-B.; Chem. Rev. 2001, 101, 757.

12. Pinheiro, S.; Pedraza, S. F.; Peralta, M. A.; Teixeira, R. C.; Farias, F. M. C.; Ferreira, V. F.; Costa, P. R. R.; Tetrahedron: Asymmetry 2002, 13, 2513.

13. Corrêa Jr., I. R.; Pilli, R. A.; Quim. Nova 2003, 26, 531.

14. Palomo, C.; Oiarbide, M.; Garcia, J. M.; Chem. Soc. Rev. 2004, 33, 65.

15. Kover, W. B.; Jones-Jr, J.; J. Braz. Chem. Soc. 1996, 7, 257.

16. Costa, P. R. R.; Ferreira, V. F.; Filho, H. C. A.; Pinheiro, S.; J. Braz. Chem. Soc. 1996, 7, 67.

17. Park, D. H.; Choi, H. J.; Lee, S-G.; J. Korean Chem. Soc. 2003, 47, 597; Seebach, D.; Wang, Z. Y.; Shen, I.; Jiang, C. S.; You, T. P.; Chin. Chem. Lett. 2000, 11, 659; Mi, A. Q.; Wang, Z. Y.; Zhang, X. M.; Fu, F. M.; Jiang, Y. Z.; Acta Chim. Sin. 1998, 56, 719; Andrés, J. M.; Martinez, M. A.; Pedroza, R.; Pérez-Encabo, A.; Synthesis 1996, 1070; Johar, P. S.; Araki, S.; Butsugan, S.; J. Chem. Soc., Perkin Trans. 1 1992, 711; Langer, W.;
Helv. Chim. Acta 1979, 62, 1701

18. Ribeiro, C. M. R.; Santos, E. de S.; Jardim, A. H. de O.; Maia, M. P.; Silva, F. C.; Moreira, A. P. D.; Ferreira, V. F.; Tetrahedron: Asymmetry 2002, 13, 1703.

19. Ribeiro, C. M. R.; Passaroto, E. N.; Brenelli, E. C. S.; J. Braz. Chem. Soc. 2001, 12, 742; Ribeiro, C. M. R.; Passaroto, E. N.; Brenelli; Tetrahedron Lett. 2001, 42, 6477.

20. Perrin, D. D.; Armarego, W. L. F.; Purification of Laboratory Chemicals, $3^{\text {rd }}$ ed., Pergamon Press: New York, 1988.

21. Ray, R.; Matterson, D. S.; Tetrahedron Lett. 1980, 21, 449.

22. Brown, H. C.; Zweifel, G.; J. Am. Chem. Soc. 1961, 83, 2544.

23. Gonçalvez, A. S.; Dissertação de Mestrado, Universidade Federal Fluminense, Brasil, 1998.

24. Parham, W. E.; Piccirilli, R. M.; J. Org. Chem. 1977, 42, 257; Parham, W. E.; Jones, L. D.; J. Org. Chem. 1976, 41, 1187; Walter, L. A.; Org. Synth. $\mathbf{1 9 5 5}, 3,737$. 